\title{
Reduced density of glutamine synthetase immunoreactive astrocytes in different cortical areas in major depression but not in bipolar I disorder
}

\author{
Hans-Gert Bernstein ${ }^{1 *}$, Gabriela Meyer-Lotz ${ }^{1}$, Henrik Dobrowolny ${ }^{1}$, Jana Bannier ${ }^{1}$, \\ Johann Steiner ${ }^{1}$, Martin Walter ${ }^{1,2}$ and Bernhard Bogerts ${ }^{1}$ \\ ${ }^{1}$ Department of Psychiatry, University of Magdeburg, Magdeburg, Germany, ${ }^{2}$ Clinical Affective Neuroimaging Laboratory, \\ University of Magdeburg, Magdeburg, Germany
}

\section{OPEN ACCESS}

Edited by:

Andrea Nistri,

Scuola Internazionale Superiore di

Studi Avanzati, Italy

Reviewed by:

Enrica Maria Petrini

Istituto Italiano di Tecnologia, Italy

Pavel Katsel,

Mount Sinai School of Medicine, USA

*Correspondence:

Hans-Gert Bernstein,

Department of Psychiatry, University of Magdeburg, Leipziger Strasse 44,

39120 Magdeburg, Germany

hans-gert.bernstein@med.ovgu.de

Received: 02 June 2015

Accepted: 03 July 2015

Published: 10 August 2015

Citation:

Bernstein H-G, Meyer-Lotz G, Dobrowolny H, Bannier J, Steiner J,

Walter M and Bogerts B (2015)

Reduced density of glutamine

synthetase immunoreactive astrocytes in different cortical areas in major depression but not in bipolar

I disorder.

Front. Cell. Neurosci. 9:273. doi: 10.3389/fncel.2015.00273
There is increasing evidence for disturbances within the glutamate system in patients with affective disorders, which involve disruptions of the glutamate-glutamine-cycle. The mainly astroglia-located enzyme glutamine synthetase (GS) catalyzes the ATPdependent condensation of ammonia and glutamate to form glutamine, thus playing a central role in glutamate and glutamine homoeostasis. However, GS is also expressed in numerous oligodendrocytes (OLs), another class of glial cells implicated in mood disorder pathology. To learn more about the role of glia-associated GS in mental illnesses, we decided to find out if numerical densities of glial cells immunostained for the enzyme protein differ between subjects with major depressive disorder, bipolar disorder (BD), and psychically healthy control cases. Counting of GS expressing astrocytes (ACs) and OLs in eight cortical and two subcortical brain regions of subjects with mood disorder $(N=14), \mathrm{BD}(N=15)$, and controls $(N=16)$ revealed that in major depression the densities of ACs were significantly reduced in some cortical but not subcortical gray matter areas, whereas no changes were found for OLs. In BD no alterations of GSimmunoreactive glia were found. From our findings we conclude that (1) GS expressing ACs are prominently involved in glutamate-related disturbances in major depression, but not in BD and (2) GS expressing OLs, though being present in significant numbers in prefrontal cortical areas, play a minor (if any) role in mood disorder pathology. The latter assumption is supported by findings of others showing that - at least in the mouse brain cortex - GS immunoreactive oligodendroglial cells are unable to contribute to the glutamate-glutamine-cycle due to the complete lack of amino acid transporters (Takasaki et al., 2010).

Keywords: major depression, bipolar disorder, cortex, glutamine synthetase, astroglia, oligodendroglia, immunocytochemistry

Abbreviations: $\mathrm{AC}(\mathrm{s})$, astrocyte(s); Aic, anterior insular cortex; ANOVA, A single-factor analysis of variance; ATP, adenosine triphosphate; BD, bipolar disorder; CNS, Central Nervous System; DLPFC, dorso-lateral prefrontal cortex; GABA, $\gamma$-Aminobutyric acid; DSM-IV; Diagnostic and Statistical Manual of Mental Disorders IVth edition; GFAP; glial fibrillary acidic protein; Glx, glutamate-glutamine-GABA complex; GS, glutamine synthetase; MANOVA, Multivariate analysis of covariance; MDD, major depression disorder; MSR, Magnetic Resonance Spectroscopy; OL(s), oligodendrocyte(s); n.a., not available; NAc, Nuc. Accumbens; pACC, pregenual anterior cingulate; PBS, Phosphate buffered saline; sACC, subgenual anterior cingulate cortex. 


\section{Introduction}

Initially, search for the possible cellular substrate of mood disorder pathology had focused on neurons (Rajkowska, 2000, 2002; Cotter et al., 2001a,b, 2002; Manji et al., 2001). However, during the last years a wave of information appeared to suggest that glial cells prominently, and in many ways, contribute to brain structural and functional changes in mood disorders. In numerous histological postmortem investigations both significantly reduced (Öngür et al., 1998; Rajkowska, 2000; Cotter et al., 2001a,b; Hamidi et al., 2004; Uranova et al., 2004; Rajkowska and Miguel-Hidalgo, 2007; Altshuler et al., 2010; Gos et al., 2013) and increased (Davis et al., 2002; Mosebach et al., 2013; Malchow et al., 2014) glial cell numbers and numerical densities have been observed in prefrontal cortex areas and limbic regions. In addition, characteristic changes in gene expression patterns and metabolic pathways of glial cells have been found in affective disorders (reviewed in Barley et al., 2009; Steiner et al., 2012; Mosebach et al., 2013; Duncan et al., 2014; Schroeter et al., 2014; Bernstein et al., 2015). Of note, each of the three glial cell classes (i.e., ACs, OLs, and microglia) appears to confer its unique contribution to the pathophysiology of affective disorders. Moreover, their impact may be different in MDD (Verkhratsky et al., 2014) and in BD, (Savitz et al., 2014; Dong and Zhen, 2015). In this context, much attention has been paid to the possible role of $\mathrm{ACs}$ in $\mathrm{MDD}$ and $\mathrm{BD}$ pathology. Being the most prevalent cell type in the human brain, ACs serve a wide range of different functions in the CNS. They play important roles in synaptic transmission, control of neuronal metabolism, sensing of brain microenvironment, maintenance of bloodbrain barrier integrity, brain defense and inflammatory processes (Sofroniew and Vinters, 2010; Teschemacher et al., 2015). Since synaptic, metabolic and inflammatory dysregulation are reported in MDD and BD (Harrison, 2002; Si et al., 2004; Kato et al., 2013; Duman, 2014; Maletic and Raison, 2014; and others), AC abnormalities may be implicated in these disorders. ACs exert influence on cerebral information processing mainly in two ways: (1) they release, by exocytosis, gliotransmitters (glutamate, D-serine, GABA and ATP, Sahlender et al., 2014), which facilitate the communication between neurons and neuron-glia crosstalk, and (2) they remove glutamate from extracellular space and provide glutamatergic and GABAergic neurons with glutamine, which they synthesize from glutamate, ammonia and ATP (Anlauf and Derouiche, 2013). Theoretically, impaired release of gliotransmitters (Van Horn et al., 2013) and/or compromised glutamate uptake and recycling by ACs might significantly contribute to anomalies of glutamatergic (and, most probably, GABAergic) neurotransmission reported in MDD and BD (for reviews, see Walter et al., 2009; Brennan et al., 2010; Gigante et al., 2012; Salvadore et al., 2012; Bernstein et al., 2013; Dou et al., 2013; Duman, 2014; Arnone et al., 2015; Pehrson and Sanchez, 2015). However, while knowledge about the possible impact of gliotransmitters on mood disorder pathology is still fragmentary (Etiévant et al., 2013), there is some good evidence in favor of impaired AC-related glutamate-glutamine cycling as an important contributing factor in affective disorders (reviewed in detail by Bernstein et al., 2013 and Rajkowska and Stockmeier, 2013). Due to its central position in the glutamate-glutamine-GABA cycle, the glutamine-synthesizing enzyme GS (aka glutamate-ammonia ligase, EC 6.3.1.2) came early into focus of research, and a number of papers have meanwhile been published about alterations in GS in MDD and $\mathrm{BD}$. In subjects with MDD a majority of studies show a decrease of cerebral GS. The expression of GS mRNA was reported to be down-regulated in the prefrontal cortex, the premotor cortex, and the amygdala of depressed suicide victims but not in suicide completers without depression (Choudary et al., 2005; Sequeira et al., 2009), whereas GS protein was found to be reduced in the anterior cingulate cortex and the orbitofrontal cortex (Choudary et al., 2005; Beasley et al., 2006; Miguel-Hidalgo et al., 2010; Rajkowska and Stockmeier, 2013). However, no alterations in cortical GS of MDD subjects were found by others (Toro et al., 2006). The implication of GS for $\mathrm{BD}$ is less well explored, and the sparse data are inconsistent. GS expression was reported unchanged (Toro et al., 2006) or decreased (Choudary et al., 2005) in the prefrontal cortex of bipolar subjects. Remarkably, all but one (Toro et al., 2006) studies on GS expression in mood disorders used biochemical techniques. Thus, little attention has yet been paid to the type of GS-expressing cells, although there is evidence that GS can also be detected in extra-astroglial localizations [i.e., in numerous OLs and even in some neurons (Takasaki et al., 2010; Iwata et al., 2013; Bernstein et al., 2014 and others)]. Thus, theoretically, disease-related alterations of GS expression might involve nonastroglial cells, too. We, therefore, counted GS expressing glial cells in ten different cortical and subcortical brain areas of subjects with $\mathrm{MDD}, \mathrm{BD}$, and psychically healthy controls and show herein that (1) significantly reduced densities of GSimmunoreactive glial cells occur in cortical areas of MDD, but not BD subjects and (2) these changes are restricted to GS expressing ACs, whereas GS expressing OLs are normal in mood disorders.

\section{Materials and Methods}

\section{Subjects}

All brains were obtained from the New Magdeburg brain collection. Case recruitment, acquisition of personal data, performance of autopsy, and handling of autoptic material were conducted in strict accordance with the Declaration of Helsinki, and have been approved by the responsible Ethical Committee of Magdeburg. Written consent was obtained from the next-ofkin. Information for clinical diagnoses was obtained from clinical records and/or structural interviews of physicians involved in treatment or relatives (Bielau et al., 2005).

Brains of 29 human subjects with mood disorders according to DSM-IV were studied. Of these individuals 14 (8 female, 6 male; mean age: $46.9 \pm 11.4$ years) had suffered from a MDD and 15 (5 female, 10 male; mean age: $53.5 \pm 10.4$ years) had a BD. Sixteen control individuals ( 9 female, 7 male; mean age: $50.4 \pm 11.0$ years) without a history of neuropsychiatric disorder were also investigated. None of the patients or controls had a history of substance abuse or alcoholism. Neuropathological 
changes due to neurodegenerative or traumatic processes were ruled out by an experienced neuropathologist as previously described (Bielau et al., 2005). These cases were matched with respect to age, gender, and autolysis time. The matching processes were done prior to all analyses. For demographical, clinical, and psychopharmacological details see Tables $\mathbf{1}$ and 2. The mean daily doses of psychotropic medication taken by patients during the last 90 lifetime days were established according to the clinical files. Since our brain bank has been established about 25 years ago, patients received tricyclic antidepressants instead of selective serotonin or noradrenalin reuptake inhibitors (for detailed considerations, see Mosebach et al., 2013).

\section{Tissue Processing}

Brains were removed within 4-96 h after death and fixed in toto in $8 \%$ phosphate-buffered formaldehyde for at least 2 months $\left(\mathrm{pH}=7.0, \mathrm{~T}=15-20^{\circ} \mathrm{C}\right)$.

Frontal and occipital poles were separated by coronal cuts $0.9 \mathrm{~cm}$ anterior to the genu and posterior to the splenium of the corpus callosum. After embedding of all parts of the brains in paraffin, serial coronal sections of the prefrontal and the middle blocks were cut $(20 \mu \mathrm{m})$ and mounted. The shrinkage factor caused by fixation and embedding of the brains was calculated by a method described previously (Bernstein et al., 1998a). The mean volume shrinkage factor for patients with affective disorders and controls was 2.21. No significant differences in the shrinkage factors among the three groups MDD, BP, and controls were found. Every 50th section was Nissl and myelin stained as described previously (Bernstein et al., 1998b).

\section{Glutamine Synthetase Immunohistochemistry}

For immunohistochemical stainings, whole brain sections were collected at intervals of about $0.2 \mathrm{~cm}$ between 1.8 and $1 \mathrm{~cm}$ rostral to the genu of the corpus callosum. The pACC, (Brodmann Area 32) and dorsolateral prefrontal (DLPFC, Brodmann Area 9) cortices were easily identifiable using the "Atlas of the Human Brain" by Mai et al. (2003). Sections containing the left and right sACC, Aic, (Brodmann area 14), and the NAc were selected at intervals of $0.2 \mathrm{~cm}$. To immunolocalize GS, we employed a well-characterized, monospecific polyclonal antiserum generated in rabbits against human GS (Prestige Antibody HPA 007316; Lot C 81287; from Sigma-Aldrich, Munich, Germany). Since different lots of the same antibody may considerably differ with regard to their staining properties (Couchman, 2009), we tested three different lots of the GS antiserum HPA 007316 (namely A42599, C81287, R04375). In our hands all three lots were of the same superior quality, and we decided to continue working with Lot C 81287. After dewaxing antigen demasking was carried out by boiling the sections for $4 \mathrm{~min}$ in $10 \mathrm{mM}$ citrate buffer ( $\mathrm{pH}$ 6.0). Thereafter, the sections were pre-incubated with methanol $/ \mathrm{H}_{2} \mathrm{O}_{2}$ to suppress endogenous peroxidases and repeatedly washed with PBS. Subsequently, the primary GS antibody was applied at a dilution of 1:500 for $72 \mathrm{~h}$ at $4^{\circ} \mathrm{C}$. Sections were then incubated with a biotinylated anti rabbit IgG (Amersham Bioscience, Buckinghamshire, GB), followed by the streptavidin horse radish complex for the application of the streptavidin-biotin technique (Amersham). The chromogen 3,3'diaminobenzidine was used to visualize the reaction product. Subsequently, ammonium nickel sulfate hexahydrate was added to enhance the immunoreaction (Bernstein et al., 2013). For control purposes, the primary antiserum was replaced by either buffer or normal serum. Further control experiments involved the application of the GS antiserum after preabsorption with GS protein (recombinant human GS, charge number CE02; from Novoprotein, Shanghai, China) as described earlier in detail (Bernstein et al., 2014). When these controls were done the investigated regions did not show any specific immunostaining.

\section{Glial Fibrillary Acidic Protein (GFAP) Immunohistochemistry}

For reasons of comparison and better delineation of cortical gray matter areas sections adjacent to GS immunostained ones were immunolabeled for GFAP. A monoclonal antibody (diluted 1:100 in PBS, from DAKO) was used. The secondary antibody was an anti-mouse peroxidase (from Biozol, Eching, Germany; dilution 1:50). The working dilution was 1:2000. Visualization was as described for GS. Controls involved replacement of the primary antiserum by either buffer or normal serum.

\section{Cell Countings}

The actual section thickness after the histological procedures was $18.9 \pm 1.0 \mu \mathrm{m}$ (mean $\pm \mathrm{SD}$ ). The optical disector cellcounting method was employed. Cell countings were performed in two coronal sections per brain area under blind conditions. A counting grid was used to define a three-dimensional box within the thickness of the section as described previously (Bernstein et al., 1998b) allowing at least $4 \mu \mathrm{M}$ guard zones at the top and bottom of the section, and to apply a direct, threedimensional counting method. Fifteen consecutive boxes per left and right cortical area were positioned, spanning the layers I and II, layer III, and another 15 spanning the layers IV, V, and VI, thus subdividing cortical gray matter regions into superficial and deep layers as proposed by Katsel et al. (2011). To count immunostained cells in the NAc we used 15 boxes per section and hemisphere. The packing densities of glial cells are noted as the number of cells $\times 10^{3} / \mathrm{mm}^{3}$. The product of the volume and glial cell density provided the total cell number estimates. The interrater reliability was about 0.87 . In a few cases we observed some local tissue damage in the brain region of interest. Cell counts of these particular regions were rigorously excluded from further calculations, which resulted in smaller cohorts than nominally indicated (for example, number of right NAc in MDD subjects: 12 instead of 14 cases).

\section{Statistical Analysis}

A single-factor analysis of variance was performed using diagnostic groups as a three-level independent variable (MDD patients versus $\mathrm{BD}$ versus non-psychiatric controls) and measured and calculated parameters were treated as dependent variables. MANOVA was performed with diagnosis and side, ie., left and right hemisphere, as independent variables (repeated measures). Effect sizes were determined for 3-group comparisons 
TABLE 1 | Demographical data of patients and controls.

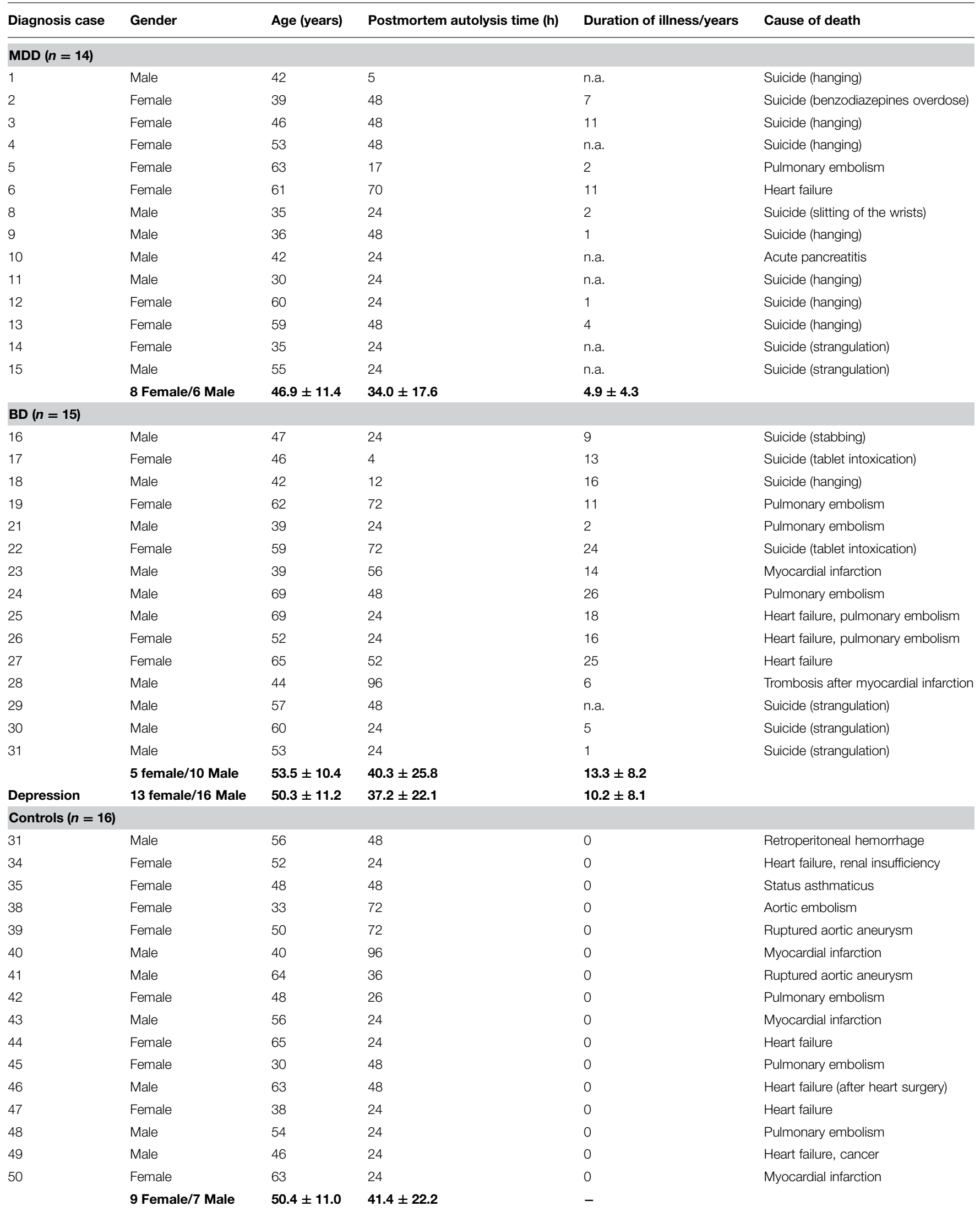


TABLE 2 | Psychopharmacological treatment.

\begin{tabular}{|c|c|c|c|c|c|}
\hline Case & $\begin{array}{l}\text { Mean antidepressiva dose } \\
\text { of the last days [mg] }\end{array}$ & $\begin{array}{l}\text { Mean neuroleptics dose of } \\
\text { the last days [mg] }\end{array}$ & $\begin{array}{l}\text { Mean benzodiazepine } \\
\text { dose of the last days [mg] }\end{array}$ & $\begin{array}{l}\text { Mean carbamazepine } \\
\text { dose of the last days [mg] }\end{array}$ & $\begin{array}{l}\text { Mean lithium dose of the } \\
\text { last days [mg] }\end{array}$ \\
\hline \multicolumn{6}{|c|}{$\operatorname{MDD}(n=14)$} \\
\hline 2 & 93 & 0 & 3 & 0 & 560 \\
\hline 3 & 124 & 109 & 0 & 0 & 0 \\
\hline 4 & 0 & 0 & 0 & 0 & 0 \\
\hline 8 & 0 & 0 & 0 & 0 & 0 \\
\hline 9 & 0 & 0 & 0 & 0 & 0 \\
\hline 10 & 200 & 200 & n.a. & n.a. & n.a. \\
\hline 11 & 100 & 100 & n.a. & n.a. & n.a. \\
\hline 12 & 100 & 440 & 0 & 0 & 0 \\
\hline 17 & 133 & 327 & 3 & 0 & 558 \\
\hline 18 & 95 & 47 & 18 & 0 & 565 \\
\hline 19 & 0 & 110 & 18 & 0 & 0 \\
\hline 21 & 0 & 280 & 0 & 0 & 0 \\
\hline 22 & 112 & 0 & 10 & 600 & 0 \\
\hline 23 & 0 & 221 & 1 & 0 & 740 \\
\hline 24 & 0 & 0 & 7 & 0 & 0 \\
\hline 25 & 0 & 0 & 2 & 0 & 280 \\
\hline 26 & 0 & n.a. & n.a. & n.a. & n.a. \\
\hline 27 & 93 & 117 & 4 & 0 & 0 \\
\hline
\end{tabular}

(major depressive disorder, BD, controls). Confounding variables including whole brain volume were primarily tested on normality by use of the Kolmogorov-Smirnov test. A post hoc Tukey HSD test was conducted to determine which variables were significantly different from each other. Pearson correlation tests were carried out to investigate effects of postmortem delay, time of fixation, illness duration, number of illness episodes, and psychotropic medication (i.e., antidepressants, neuroleptics, benzodiazepines, and lithium) on data. In addition, emphasis was given to suicide as possible confounding factor. $P$-values less than 0.05 were deemed to be statistically significant.

\section{Results}

\section{Qualitative Observations Glutamine Synthetase Immunostaining Astrocytes}

Glutamine synthetase -immunoreactive ACs were abundantly present in the cerebral cortex and the NAc. Their morphology was remarkably consistent. Astrocytic somata and processes were prominently stained for GS. In addition, an intense immunostaining was observed in the neuropil. Numerous blood vessels were surrounded by GS-immunoreactive AC endfeet.

\section{Oligodendrocytes}

Gray matter GS-immunoreactive OLs were easily identifiable based on their typical morphology. The immunoreaction was confined to the cell somata. Short processes were only infrequently immunolabeled. Immunopositive OLs were fairly uniformly distributed throughout prefrontal cerebral cortex. However, in the AiC and the NAc GS-expressing OLs were relatively rarely found. Therefore, we did not count them separately in the latter two brain regions. Examples for the immunolocalization of GS in ACs and OLs are given in Figures 1A-I. Figure 1J shows a control reaction.

\section{GFAP Immunostaining}

In all cortical areas GFAP protein was expressed in a majority of ACs. Their distribution showed a laminar pattern. The highest package density of GFAP was found in layers I (where ACs abut at the pial surface of the brain, Miguel-Hidalgo et al., 2010) and 


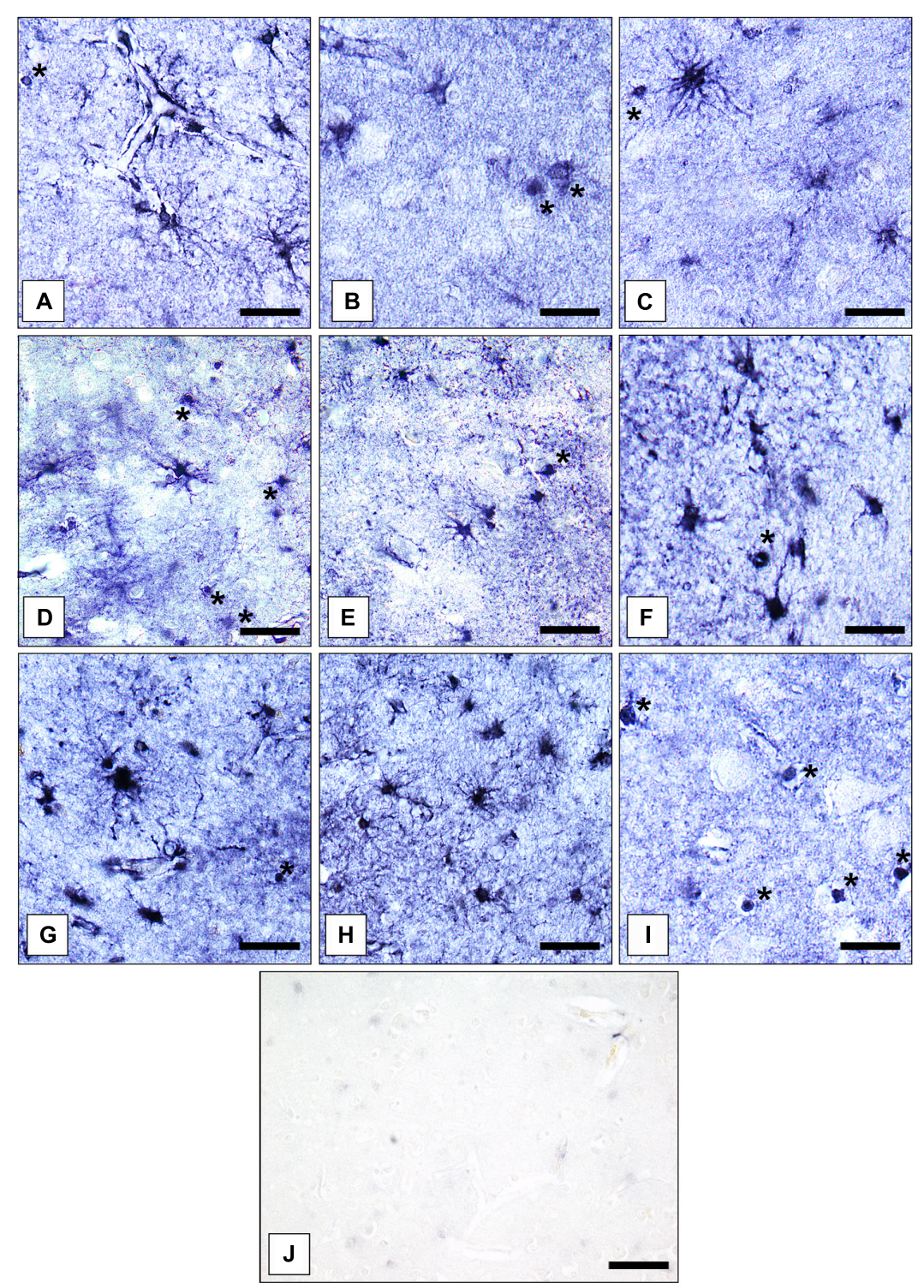

FIGURE 1 | Immunolocalization of GS in cortical and subcortical human brain glial cells in MDD, BD, and controls. (A) GS-immunoreactive ACs and OLs (asterisk) in the pACC (control case). Bar $=20 \mu \mathrm{m}$. (B) GS-immunoreactive ACs and OLs (asterisks) in the pACC (MDD subject). Bar $=20 \mu \mathrm{m}$.

(C) GS-immunoreactive ACs and OLs (asterisk) in the pACC (BD subject). Bar $=20 \mu \mathrm{m}$. (D) GS-immunoreactive ACs and OLs (asterisks) in the sACC (control case). Bar $=20 \mu \mathrm{m}$.(E) GS-expressing ACs and OLs (asterisk) in the
sACC (MDD subject). Bar $=20 \mu \mathrm{m}$.(F) GS-expressing ACs and OLs (asterisk) in the DLPFC (BD subject). Bar $=20 \mu \mathrm{m}$. (G) GS-immunopositive ACs in the AiC (control case). Bar $=20 \mu \mathrm{m}$. (H) GS-expressing ACs in the NAc (control case). Bar $=24 \mu \mathrm{m}$. (I) GS-immunoreactive OLs (asterisks) in the pACC (control case). Bar $=20 \mu \mathrm{m}$. (J) Specificity control reaction. After preabsorption of the primary antiserum with recombinant GS protein no specific immunostaining is visible. Bar $=30 \mu \mathrm{m}$.
II. In the NAc an even distribution of GFAP immunolabeled cell elements was observed. OLs did not express GFAP.

\section{Quantitative Estimates}

We could replicate our own findings (Bernstein et al., 2014) as well as results previously published by others (Toro et al., 2006) that there is a higher density of GS-expressing cells in superficial cortical layers I-III than in deeper cortical layers IVVI. It is therefore justified to count superficial and deeper layers separately.
In subjects with mood disorder significantly reduced numerical densities of GS immunoreactive ACs were found the DLPFC (left side, layers I-III, $p=0.029 ; F=4.487$ and right side, layers I-III; $p=0.046 ; F=3.647$ ), sACC (left side, layers I-III, $p=0.021 ; F=4.304$ ), AiC (left side, layers I-III, $p=0.002$; $F=7.893$; right side, layers I-III, $p=0.001 ; F=9.789$; ride side, layers IV-VI, $p=0.015 ; F=5.136$ ). No significant changes were detectable in the pACC and the NAc. All but one (DLPCC right side, layers I-III) significant changes survived Tukey's HSD post hoc tests. Notably, no significant alterations in the numerical 
densities of OLs were found. Results of the morphometric analyses are shown in Figures 2-6.

\section{MDD vs. controls}

Compared with controls a significant reduction in the numerical densities of GS-immunopositive ACs was found in MDD in five of the 10 cortical and subcortical brain regions studied: DLPFC (left side, layers I-III, $p=0.024$ ), sACC (left side, layers I-III, $p=0.018$ ); AiC (left side, layers I-III; $p=0.002$; right side, layers I-III; $p=0.001 ; F=9.962$; right side, layers IV-VI, $p=0.012$ ).

\section{BD vs. controls}

In $\mathrm{BD}$ cases the numerical densities of GS-expressing ACs and OLs did not significantly differ from those of controls.

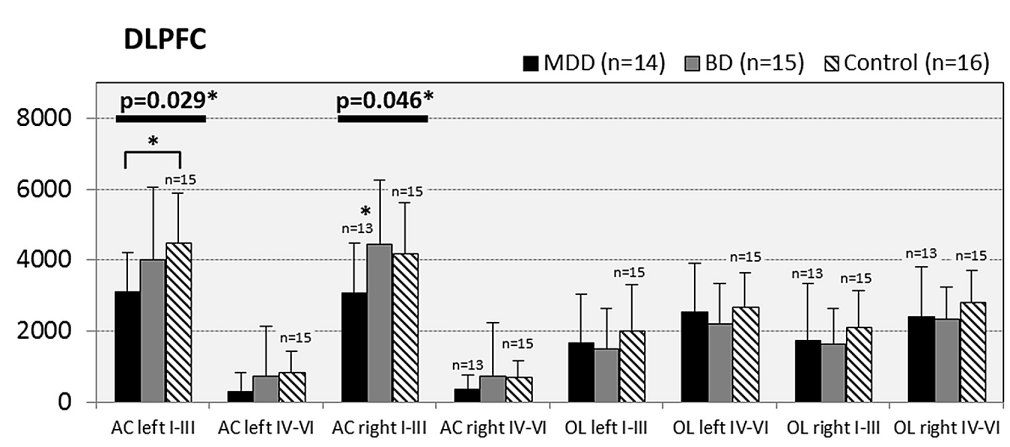

FIGURE 2 | Numerical densities of GS-expressing glial cells (ACs and OLs) in the DLPFC of subjects with MDD, BD, and controls. Compared with controls the density of GS-expressing ACs is significantly reduced in MDD cases (AC left I-III, $p=0.029$; AC right I-III, $p=0.046$ ).

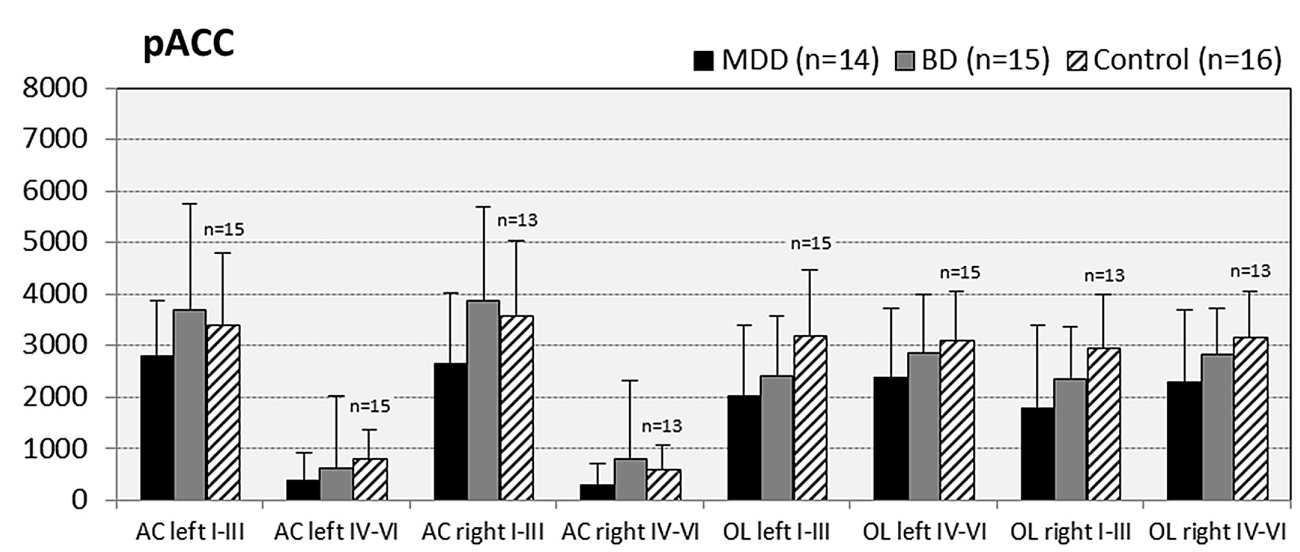

FIGURE 3 | Numerical densities of GS-expressing glial cells (ACs and OLs) in the pACC of subjects with MDD, BD, and controls. No significant alterations were found in this brain region.

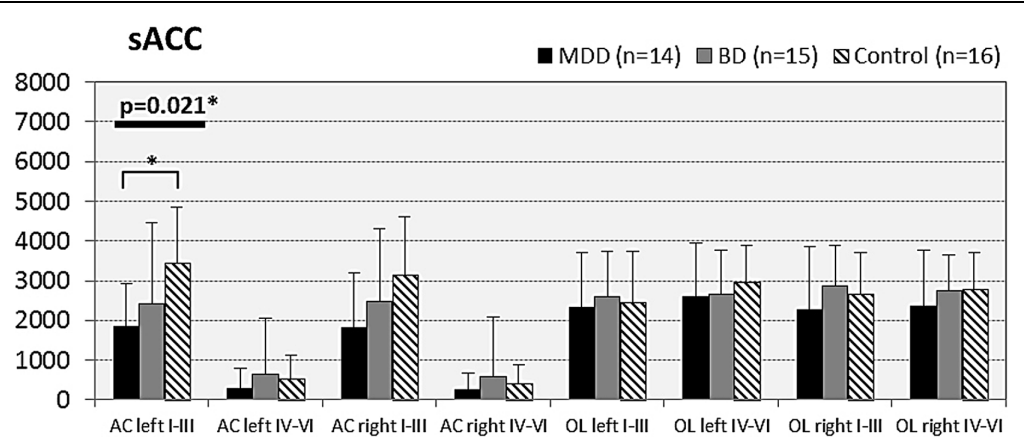

FIGURE 4 | Numerical densities of GS-expressing glial cells (ACs and OLs) in the sACC of subjects with MDD, BD, and controls. Compared with controls the density of GS-expressing ACs is significantly reduced in MDD cases (AC left I-III, $p=0.021$ ). 

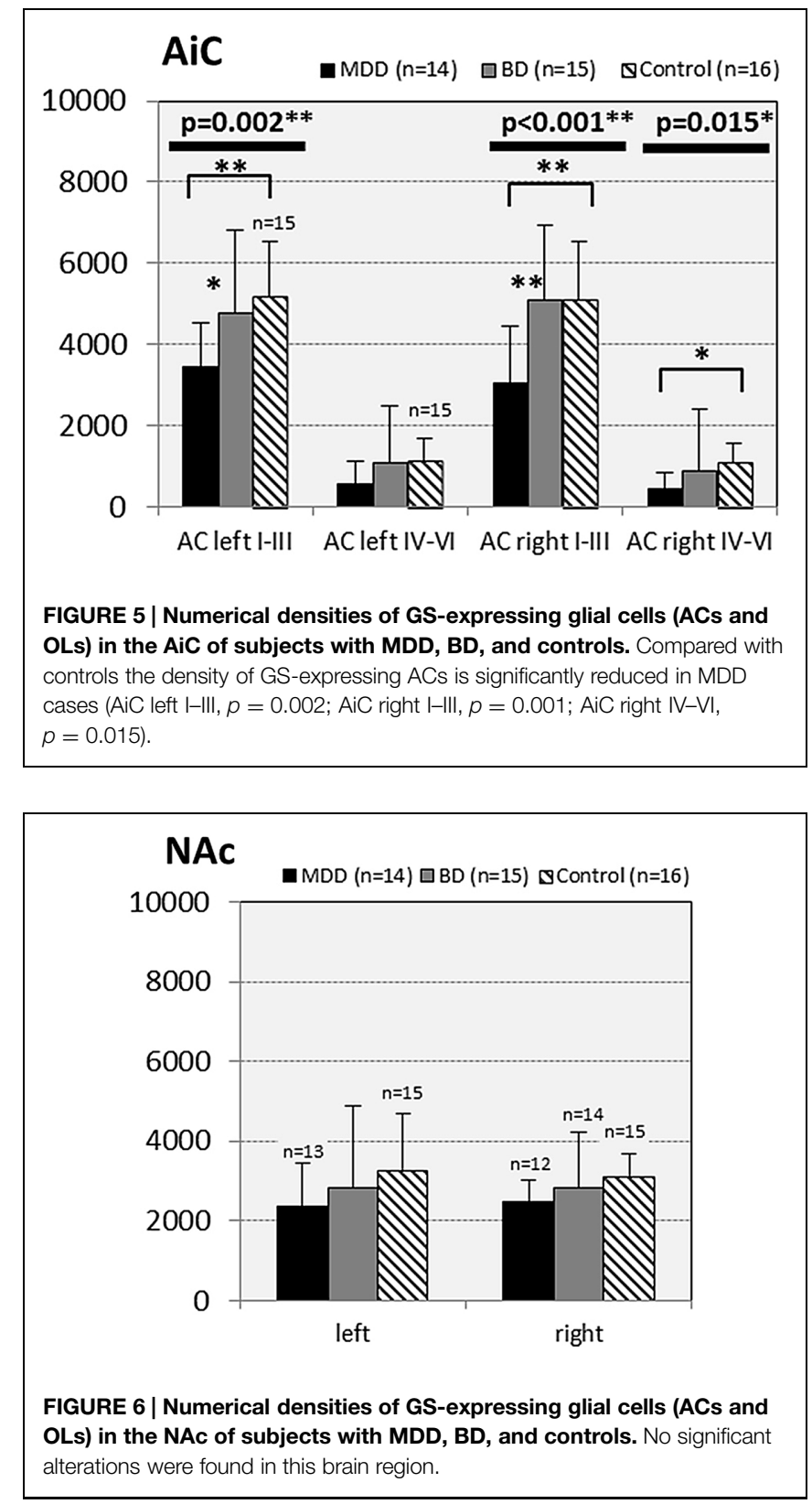

\section{MDD vs. BD}

Compared with BD cases subjects with MDD showed significantly decreased densities of GS-immunopositive ACs in the DLPFC (right side, layers I-III, $P=0.047$ ), in the AiC (left side, layers I-III, $p=0.024$; right side, layers IV.IV; $p=0.001$ ).

\section{Impact of Completed Suicide on Cell Densities}

Since there are reports showing that GS expression is altered in brains of suicide victims with and without mood disorder (Kim et al., 2007; Sequeira et al., 2009; Bernstein et al., 2013) we next analyzed the influence of the confounding factor "death by suicide" on the densities of GS immunoreactive ACs in the brain regions under study. For this purpose we divided MDD and $\mathrm{BD}$ cases into subgroups of suicide victims (MDD: $N=11$;
BD: $N=7$ ) and depressed subjects who died of natural causes (MDD: $N=3$; BD: $N=8$ ). With regard to MDD cases in none of the brain regions there appeared significant differences in the densities of GS-expressing cells between depressed subjects dying by suicide and non-suicidal individuals with MDD. The same holds true for subjects with BD: Suicidal and non-suicidal BD cases did not significantly differ with regard to glial cell densities in any of the regions studied, as exemplified for the AiC in Figure 7. It should be emphasized, however, that the subgroup of non-suicidal subjects with MDD is too small $(N=3)$ to come to far-reaching conclusions from these data.

\section{The Influence of Other Confounding Factors}

Analysis of the potential confounding factors on the test results revealed no significant influence of age, gender, duration of disease, or psychotropic medication. Especially the lack of correlation between the age and the density of GS-expressing ACs is interesting, because Rajkowska and Stockmeier (2013) have hypothesized the glial pathology appears to apply mostly to younger and middle age subjects with MDD ( $<60$ years of age).

\section{Possible Influence of Hemispheric Asymmetry on GS Cell Distribution in Human Brain}

There is evidence in the for structural, functional, and physiological asymmetries in the two hemispheres of human brain (comprehensively reviewed in Jayasundar and Raghunathan, 1997), which involve aspects of glutamate and GABA metabolism in health and mood disorder (MDD: Pfleiderer et al., 2003; Bajbouj et al., 2006; Gos et al., 2012; BD: Gos et al., 2012; Xu et al., 2013). The latter might in part result from left-right hemispheric differences in the distribution of GS immunoreactivity (Bernstein et al., 2013), Unfortunately, knowledge about the catalytic activity and distribution of human brain GS comes mainly from studies of only one (namely the left) brain hemisphere (Burbaeva et al., 2003; Rajkowska and Miguel-Hidalgo, 2007; Miguel-Hidalgo et al., 2010). To exclude a possible left-right asymmetry of GS, we counted left and right hemispheres separately. No evidence was found for a significant left-right asymmetry, neither in controls, nor in subjects with affective disorder.

\section{Discussion}

Major depression disorder and $\mathrm{BD}$ are serious mental illnesses with multifactorial pathophysiologic characteristics. The past years have witnessed a remarkable extension of our understanding of the neurobiology of affective disorders, adding the glutamatergic and the GABAergic hypotheses to "classical" monoaminergic theories of mood dysregulation (Delgado, 2000; Skolnick et al., 2009; Catena-Dell'Osso et al., 2013; Dou et al., 2013; Hertz et al., 2014; Pehrson and Sanchez, 2015; Schitine et al., 2015). Besides other indicators of compromised amino acidergic neurotransmission in depression, two large meta-analyses of neuroimaging findings have demonstrated decreased levels of the Glx in adult MDD patients, whereas in the brains of $\mathrm{BD}$ patients increased, decreased, and unchanged 


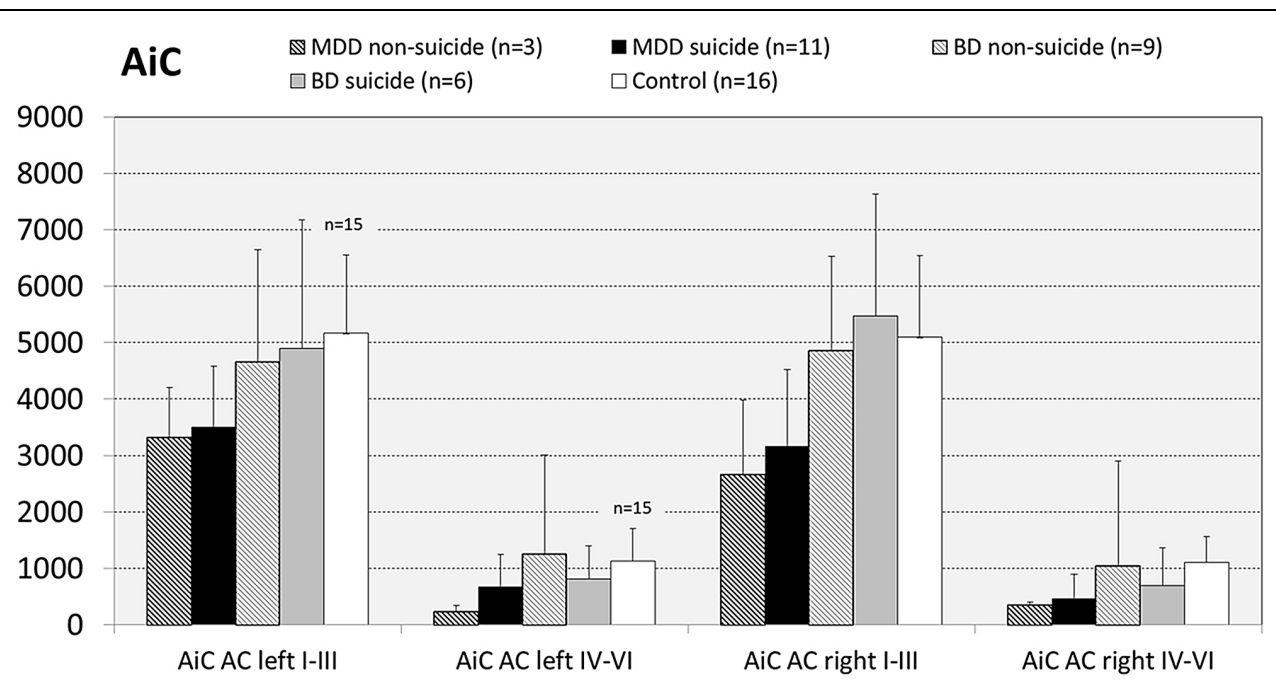

FIGURE 7 | Numerical densities of GS-expressing glial cells in the AiC of suicide completers with MDD, non-suicide completers with MDD, suicide completers with BD, non-suicide completers with BD, and controls. No significant alterations were found.

glutamate and reduced glutamine levels have been measured (Yüksel and Öngür, 2010; Gigante et al., 2012; Luykx et al., 2012). Moreover, the NMDA receptor antagonist ketamine has been shown in clinical trials to act as a rapid antidepressant in MDD (for recent considerations, see DeWilde et al., 2015). The acceptance of a crucial role of glutamate in the pathology of mental disorders entails the obligatory implication of glial cells (Arnone et al., 2015). Being part of the glutamatergic tripartite synapse, intact ACs are central to a proper functioning of glutamatergic neurotransmission, and imbalances in the neuronAC communication at the synaptic level might significantly contribute to affective disorders, such as mania and depression (Mitterauer, 2015; Thompson et al., 2015). The enzyme GS, which is highly expressed in, but in its expression not restricted to, ACs, is required to synthesize the non-toxic glutamine from the re-uptake of either glutamate or GABA. Besides, in human brain an immunologically and enzymatically very closely related "sister" enzyme of (GS-like protein) of unknown cellular localization was found (Boksha et al., 2000), which shows altered expressions in schizophrenia and Alzheimer's disease (Burbaeva et al., 2003; Burbaeva et al., 2014). The possible implication of GS-like enzyme for mood disorders is unexplored, however. Keeping this in mind when designing this study, we morphometrically analyzed GS-immunoreactive glial cells located in various cortical and subcortical gray matter areas of subjects with MDD and BD, thereby counting, wherever possible, ACs and OLs separately. Significantly reduced densities of GS immunopositive ACs were found in the prefrontal areas DLPFC and sACC as well as in the AiC of subjects with MDD. Remarkably, the densities of GS-immunopositive OLs were normal in all regions studied, although a significant loss of perineuronal OLs was found in prefrontal cortex sublayers IIIa, b, and c in mood disorder by others (Vostrikov et al., 2007). When carefully analyzing cortical GS-expressing OLs in mice, Takasaki et al. (2010) found that the enzyme protein is mainly expressed in perineuronal OLs, with roughly half of them being GS-immunopositive. However, these GS-immunoreactive OLs lack plasmalemmal glutamate transporters GLAST and GLT-1, thus apparently being unable to participate in the glutamateglutamine cycle. The reason why these cells even so express GS remains enigmatic, but is seemingly not directly related to glutamatergic synaptic neurotransmission (possibly playing a role in cellular ammonia clearance and/or certain metabolic support to the associating cortical neurons, Takasaki et al., 2010; Bernstein et al., 2014). Thus, our data clearly show a cell-type specific reduction in cortical GS expression in individuals with MDD, which cannot be revealed when analyzing total GS protein in brain samples with biochemical methods. Interestingly, we found no indication for altered densities of GS-expressing ACs in the NAc, although this brain region plays a central role in MDD (Thompson et al., 2015) and has therefore been chosen as a target structure for deep brain stimulation in cases of therapy-resistant MMD (Bewernick et al., 2012). Although ACs play a critical role in controlling the excitability of NAc neurons via activation of glutamatergic receptors (Fellin et al., 2007; Thompson et al., 2015), their possible implication for "human" depression" yet is poorly explored. However, the number of ACs in the NAc was found to be unchanged in a developmental "toxic stress" model of depression (Shende et al., 2015).

Unlike in MDD, no alterations in the density of GS-expressing glial cells were found in BD cases. This is in agreement with earlier findings by Toro et al. (2006), but in contrast to reduced GS expression in prefrontal area of BD subjects as reported by Choudary et al. (2005). Two possible conclusions may be drawn from our data: either (1) glia associated-GS is normal, because GS does not play a prominent role in the pathophysiology of $\mathrm{BD}$, and/or (2) GS expression was "normalized" (most probably up-regulated) by long-term treatment of the disease. Given the latter possibility, two putative factors might have increased GS expression in brains of BD subjects: administration of lithium 
(Kalkman, 2011) and treatment with antidepressants (Hashioka et al., 2013; Liu et al., 2015). Our calculations, however, did not reveal significant positive correlations of numerical densities of GS expressing glial cells and either of the aforementioned two factors. Hence we tend to believe that GS is not a major contributing factor to $\mathrm{BD}$ neuropathology. It should be stated, however, that disease-related alterations in the cerebral expression of GS do not always exert a direct influence on the glutamate-glutamine-GABA ratio. So, for example, a strong upregulation of GS protein was reported for the anterior cingulate cortex of female but not male individuals with schizophrenia (Martins-de-Souza et al., 2010), whereas no such gender-specific change in the Glx ratio was found in schizophrenia patients by MRS (Rowland et al., 2013). Thus, further studies are clearly needed to learn more about the impact of GS on glutamate metabolites in the brain.

\section{Limitations of the Study}

Since a major limitation of post-mortem studies is underpowered sample size, we have tried to increase the three cohorts (controls, $N=16$; $\mathrm{BD}, N=15, \mathrm{MDD}, N=14)$. A consequence from doing so is to accept the lack of data on the cumulative antidepressant exposure of some patients with MDD and BD. A further limitation of the this study is that, as in all immunocytochemistry-based morphometric studies, it cannot be said with ultimate certainty, whether the observed disease-related changes are due to glial cell loss, or to reduced intracellular expression of the protein (below the detection threshold of the method) in still existing cells, or both. Lastly, a limitation

\section{References}

Altshuler, L. L., Abulseoud, O. A., Foland-Ross, L., Bartzokis, G., Chang, S., Mintz, J., et al. (2010). Amygdala astrocyte reduction in subjects with major depression but not bipolar disorder. Bipolar Disord. 1, 451-549. doi: 10.1111/j.1399-5618.2010.00838.x

Anlauf, E., and Derouiche, A. (2013). Glutamine synthetase as an astrocytic marker: its cell type and vesicle localization. Front. Endocrinol. 4:144. doi: 10.3389/fendo.2013.00144

Arnone, D., Mumuni, A. N., Jauhar, S., Condon, B., and Cavanagh, J. (2015). Indirect evidence of selective glial involvement in glutamate-based mechanisms of mood regulation in depression: meta-analysis of absolute prefrontal neuro-metabolic concentrations. Eur. Neuropsychopharmacol. doi: 10.1016/j.euroneuro.2015.04.016 [Epub ahead of print].

Bajbouj, M., Lisanby, S. H., Lang, U. E., Danker-Hopfe, H., Heuser, I., and Neu, P. (2006). Evidence for impaired cortical inhibition in patients with unipolar major depression. Biol. Psychiatry 59, 395-400. doi: 10.1016/j.biopsych.2005.07.036

Barley, K., Dracheva, S., and Byne, W. (2009). Subcortical oligodendrocyteand astrocyte-associated gene expression in subjects with schizophrenia, major depression and bipolar disorder. Schizophr. Res. 112, 54-64. doi: 10.1016/j.schres.2009.04.01

Beasley, C. L., Pennington, K., Behan, A., Wait, R., Dunn, M. J., and Cotter, D. (2006). Proteomic analysis of the anterior cingulate cortex in the major psychiatric disorders: evidence for disease-associated changes. Proteomics 6, 3414-3425. doi: 10.1002/pmic.200500069

Bernstein, H. G., Bannier, J., Meyer-Lotz, G., Steiner, J., Keilhoff, G., Dobrowolny, H., et al. (2014). Distribution of immunoreactive glutamine synthetase in the adult human and mouse brain. Qualitative and quantitative may arise from the fact that the decreased expression of GS protein does not necessarily mean reduced activity of the enzyme. Unfortunately, it is impossible to reveal the catalytic activity of the AC-located GS enzyme because of the lack of a specific enzyme histochemical method for GS.

\section{Conclusion}

In $\mathrm{MDD}$ but not in $\mathrm{BD}$ there is a glia cell-type specific (astroglial) reduction of cortical GS protein expression, which might constitute a cellular correlate of lower cortical Glx levels reported for subjects with MDD in MSR studies.

\section{Author Contributions}

H-GB analyzed the data, researched, wrote, and edited the manuscript. GM-L analyzed the data. HD carried out statistical calculations and contributed to photography. JB analyzed the data. JS wrote, and edited the manuscript. MW wrote and edited the manuscript. BB wrote and edited the manuscript.

\section{Acknowledgments}

We are grateful to Bianca Jerzykiewicz for excellent technical assistance. This research project was financially supported by SFB 779, project number A06 of the Deutsche Forschungsgemeinschaft (Germany).

observations with special emphasis on extra-astroglial protein localization. J. Chem. Neuroanat. 61-62, 33-50. doi: 10.1016/j.jchemneu.2014.07.003

Bernstein, H. G., Krell, D., Baumann, B., Danos, P., Falkai, P., Diekmann, S., et al. (1998a). Morphometric studies of the entorhinal cortex in neuropsychiatric patients and controls: clusters of heterotopically displaced lamina II neurons are not indicative of schizophrenia. Schizophr. Res. 33, 125-132. doi: 10.1016/S0920-9964(98)00071-1

Bernstein, H. G., Stanarius, A., Baumann, B., Henning, H., Krell, D., Danos, P., et al. (1998b). Nitric oxide synthase-containing neurons in the human hypothalamus: reduced number of immunoreactive cells in the paraventricular nucleus of depressive patients and schizophrenics. Neuroscience 83, 867-875. doi: 10.1016/S0306-4522(97)00461-2

Bernstein, H. G., Steiner, J., Guest, P. C., Dobrowolny, H., and Bogerts, B. (2015). Glial cells as key players in schizophrenia pathology: recent insights and concepts of therapy. Schizophr. Res. 161, 4-18. doi: 10.1016/j.schres.2014. 03.035

Bernstein, H. G., Tausch, A., Wagner, R., Steiner, J., Seeleke, P., Walter, M., et al. (2013). Disruption of glutamate-glutamine-GABA cycle significantly impacts on suicidal behaviour: survey of the literature and own findings on glutamine synthetase. CNS Neurol. Disord. Drug Targets 12, 900-913. doi: $10.2174 / 18715273113129990091$

Bewernick, B. H., Kayser, S., Sturm, V., and Schlaepfer, T. E. (2012). Long-term effects of nucleus accumbens deep brain stimulation in treatment-resistant depression: evidence for sustained efficacy. Neuropsychopharmacology 37, 1975-1985. doi: 10.1038/npp.2012.44

Bielau, H., Trübner, K., Krell, D., Agelink, M. W., Bernstein, H. G., Stauch, R., et al. (2005). Volume deficits of subcortical nuclei in mood disorders A postmortem study. Eur. Arch. Psychiatry Clin. Neurosci. 255, 401-412. doi: 10.1007/s00406005-0581-y 
Boksha, I. S., Tereshkina, E. B., and Burbaeva, G. S. (2000). Glutamine synthetase and glutamine synthetase-like protein from human brain: purification and comparative characterization. J. Neurochem. 75, 2574-2582. doi: 10.1046/j.1471-4159.2000.0752574.x

Brennan, B. P., Hudson, J. I., Jensen, J. E., McCarthy, J., Roberts, J. L., Prescot, A. P., et al. (2010). Rapid enhancement of glutamatergic neurotransmission in bipolar depression following treatment with riluzole. Neuropsychopharmacology 35, 834-846. doi: 10.1038/npp.2009.191

Burbaeva, G. Sh., Boksha, I. S., Tereshkina, E. B., Savushkina, O. K., Prokhorova, T. A., and Vorobyeva, E. A. (2014). Glutamate and GABA-metabolizing enzymes in post-mortem cerebellum in Alzheimer's disease: phosphateactivated glutaminase and glutamic acid decarboxylase. Cerebellum 13, 607615. doi: 10.1007/s12311-014-0573-4

Burbaeva, G. Sh., Boksha, I. S., Turishcheva, M. S., Vorobyeva, E. A., Savushkina, O. K., and Tereshkina, E. B. (2003). Glutamine synthetase and glutamate dehydrogenase in the prefrontal cortex of patients with schizophrenia. Prog. Neuropsychopharmacol. Biol. Psychiatry. 27, 675-680. doi: 10.1016/S02785846(03)00078-2

Catena-Dell'Osso, M., Fagiolini, A., Rotella, F., Baroni, S., and Marazziti, D. (2013). Glutamate system as target for development of novel antidepressants. CNS Spectr. 18, 188-198. doi: 10.1017/S1092852912000971

Choudary, P. V., Molnar, M., Evans, S. J., Tomita, H., Li, J. Z., and Vawter, M. P. (2005). Altered cortical glutamatergic and GABAergic signal transmission with glial involvement in depression. Proc. Natl. Acad. Sci. U.S.A. 102, 15653-15658. doi: 10.1073/pnas.0507901102

Cotter, D., Mackay, D., Chana, G., Beasley, C., Landau, S., and Everall, I. P. (2002). Reduced neuronal size and glial cell density in area 9 of the dorsolateral prefrontal cortex in subjects with major depressive disorder. Cereb. Cortex 12, 386-394. doi: 10.1093/cercor/12.4.386

Cotter, D., Mackay, D., Landau, S., Kerwin, R., and Everall, I. (2001a). Reduced glial cell density and neuronal size in the anterior cingulate cortex in major depressive disorder. Arch. Gen. Psychiatry 58, 545-553. doi: 10.1001/archpsyc.58.6.545

Cotter, D. R., Pariante, C. M., and Everall, I. P. (2001b). Glial cell abnormalities in major psychiatric disorders: the evidence and implications. Brain Res. Bull. 55, 585-595. doi: 10.1016/S0361-9230(01)00527-5

Couchman, J. R. (2009). Commercial antibodies: the good, bad, and really ugly. J. Histochem. Cytochem. 57, 7-8. doi: 10.1369/jhc.2008.952820

Davis, S., Thomas, A., Perry, R., Oakley, A., Kalaria, R. N., and O’Brien, J. T. (2002). Glial fibrillary acidic protein in late life major depressive disorder: an immunocytochemical study. J. Neurol. Neurosurg. Psychiatry 73, 556-560. doi: 10.1136/jnnp.73.5.556

Delgado, P. L. (2000). Depression: the case for a monoamine deficiency. J. Clin. Psychiatry 61(Suppl. 6), 7-11.

DeWilde, K. E., Levitch, C. F., Murrough, J. W., Mathew, S. J., and Iosifescu, D. V. (2015). The promise of ketamine for treatment-resistant depression: current evidence and future directions. Ann. N. Y. Acad. Sci. 1345, 47-58. doi: $10.1111 /$ nyas. 12646

Dong, X. H., and Zhen, X. C. (2015). Glial pathology in bipolar disorder: potential therapeutic implications. CNS Neurosci. Ther. 21, 393-397. doi: $10.1111 / \mathrm{cns} .12390$

Dou, W., Palomero-Gallagher, N., van Tol, M. J., Kaufmann, J., Zhong, K., Bernstein, H. G., et al. (2013). Systematic regional variations of GABA, glutamine, and glutamate concentrations follow receptor fingerprints of human cingulate cortex. J. Neurosci. 33, 12698-12704. doi: 10.1523/JNEUROSCI.175813.20

Duman, R. S. (2014). Neurobiology of stress, depression, and rapid acting antidepressants: remodeling synaptic connections. Depress. Anxiety 31, 291296. doi: 10.1002/da.22227

Duncan, L. E., Holmans, P. A., Lee, P. H., O’Dushlaine, C. T., Kirby, A. W., Smoller, J. W., et al. (2014). Pathway analyses implicate glial cells in schizophrenia. PLOS ONE 9:e89441. doi: 10.1371/journal.pone.00 89441

Etiévant, A., Lambás-Señas, L., Scarna, H., Lucas, G., and Haddjeri, N. (2013). Astrocytes and gliotransmitters: new players in the treatment of major depression? Curr. Drug Targets 14, 1295-1307. doi: $10.2174 / 13894501113149990197$
Fellin, T., D’Ascenzo, M., and Haydon, P. G. (2007). Astrocytes control neuronal excitability in the nucleus accumbens. ScientificWorldJournal 7, 89-97. doi: 10.1100/tsw.2007.195

Gigante, A. D., Bond, D. J., Lafer, B., Lam, R. W., Young, L. T., and Yatham, L. N. (2012). Brain glutamate levels measured by magnetic resonance spectroscopy in patients with bipolar disorder: a meta-analysis. Bipolar Disord. 14, 478-487. doi: 10.1111/j.1399-5618.2012.01033.x

Gos, T., Schroeter, M. L., Lessel, W., Bernstein, H. G., Dobrowolny, H., Schiltz, K., et al. (2013). S100B-immunopositive astrocytes and oligodendrocytes in the hippocampus are differentially afflicted in unipolar and bipolar depression: a postmortem study. J. Psychiatr. Res. 47, 1694-1699. doi: 10.1016/j.jpsychires.2013.07.005

Gos, T., Steiner, J., Bielau, H., Dobrowoln, H., Günther, K., Mawrin, C., et al. (2012). Differences between unipolar and bipolar I depression in the quantitative analysis of glutamic acid decarboxylase-immunoreactive neuropil. Eur. Arch. Psychiatry Clin. Neurosci. 262, 647-655. doi: 10.1007/s00406-012-0315-x

Hamidi, M., Drevets, W. C., and Price, J. L. (2004). Glial reduction in amygdala in major depressive disorder is due to oligodendrocytes. Biol. Psychiatry 55, 563-569. doi: 10.1016/j.biopsych.2003.11.006

Harrison, P. J. (2002). The neuropathology of primary mood disorder. Brain 125, 1428-1449. doi: 10.1093/brain/awf149

Hashioka, S., Miyaoka, T., Wake, R., Furuya, M., and Horiguchi, J. (2013). Glia: an important target for anti-inflammatory and antidepressant activity. Curr. Drug Targets 14, 1322-1328. doi: 10.2174/13894501113146660214

Hertz, L., Song, D., Li, B., Du, T., Xu, J., Gu, L., et al. (2014). Signal transduction in astrocytes during chronic or acute treatment with drugs (SSRIs, antibipolar Drugs, GABA-ergic drugs, and benzodiazepines) ameliorating mood disorders. J. Signal Transduct. 2014:593934. doi: 10.1155/2014/593934

Iwata, K., Café-Mendes, C. C., Schmitt, A., Steiner, J., Manabe, T., Matsuzaki, H., et al. (2013). The human oligodendrocyte proteome. Proteomics 13, 3548-3553. doi: 10.1002/pmic.201300201

Jayasundar, R., and Raghunathan, P. (1997). Evidence for left-right asymmetries in the proton MRS of brain in normal volunteers. Magn. Reson. Imaging 15, 223-234. doi: 10.1016/S0730-725X(96)00342-6

Kalkman, H. O. (2011). Circumstantial evidence for a role of glutamine-synthetase in suicide. Med. Hypotheses 76, 905-907. doi: 10.1016/j.mehy.2011.03.005

Kato, T. A., Watabe, M., and Kanba, S. (2013). Neuron-glia interaction as a possible glue to translate the mind-brain gap: a novel multi-dimensional approach toward psychology and psychiatry. Front. Psychiatry 4:139. doi: 10.3389/fpsyt.2013.00139

Katsel, P., Byne, W., Roussos, P., Tan, W., Siever, L., and Haroutunian, V. (2011). Astrocyte and glutamate markers in the superficial, deep and white matter layers of the anterior cingulate gyrus in schizophrenia. Neuropsychopharmacology 36, 1171-1177. doi: 10.1038/npp.2010.252

Kim, S., Choi, K. H., Baykiz, A. F., and Gershenfeld, H. K. (2007). Suicide candidate genes associated with bipolar disorder and schizophrenia: an exploratory gene expression profiling analysis of post-mortem prefrontal cortex. BMC Genomics 8:413. doi: 10.1186/1471-2164-8-413

Liu, Z., Song, D., Yan, E., Verkhratsky, A., and Peng, L. (2015). Chronic treatment with anti-bipolar drugs suppresses glutamate release from astroglial cultures. Amino Acids 47, 1045-1051. doi: 10.1007/s00726-015-1936-y

Luykx, J. J., Laban, K. G., van den Heuvel, M. P., Boks, M. P., Mandl, R. C., Kahn, R. S., et al. (2012). Region and state specific glutamate downregulation in major depressive disorder: a meta-analysis of (1)H-MRS findings. Neurosci. Biobehav. Rev. 36, 198-205. doi: 10.1016/j.neubiorev.2011.05.014

Mai, J. K., Paxinos, G., and Assheuer, J. K. (2003). Atlas of the Human Brain. Amsterdam: Elsevier Academic Press.

Malchow, B., Strocka, S., Frank, F., Bernstein, H. G., Steiner, J., SchneiderAxmann, T., et al. (2014). Stereological investigation of the posterior hippocampus in affective disorders. J. Neural Transm. doi: 10.1007/s00702-0141316-x [Epub ahead of print].

Maletic, V., and Raison, C. (2014). Integrated neurobiology of bipolar disorder. Front. Psychiatry 5:98. doi: 10.3389/fpsyt.2014.00098

Manji, H. K., Drevets, W. C., and Charney, D. S. (2001). The cellular neurobiology of depression. Nat. Med. 7, 541-547. doi: 10.1038/87865

Martins-de-Souza, D., Schmitt, A., Röder, R., Lebar, M., Schneider-Axmann, T., Falkai, P., et al. (2010). Sex-specific proteome differences in the anterior 
cingulate cortex of schizophrenia. J. Psychiatr. Res. 44, 989-991. doi: 10.1016/j.jpsychires.2010.03.003

Miguel-Hidalgo, J. J., Waltzer, R., Whittom, A. A., Austin, M. C., Rajkowska, G., and Stockmeier, C. A. (2010). Glial and glutamatergic markers in depression, alcoholism, and their comorbidity. J. Affect. Disord. 127, 230-240. doi: 10.1016/j.jad.2010.06.003

Mitterauer, B. J. (2015). Balancing and imbalancing effects of astrocytic receptors in tripartite synapses. Common pathophysiological model of mental disorders and epilepsy. Med. Hypotheses 84, 315-320. doi: 10.1016/j.mehy.2015.01.025

Mosebach, J., Keilhoff, G., Gos, T., Schiltz, K., Schoeneck, L., Dobrowolny, H., et al. (2013). Increased nuclear Olig1-expression in the pregenual anterior cingulate white matter of patients with major depression: a regenerative attempt to compensate oligodendrocyte loss? J. Psychiatr. Res. 47, 1069-1079. doi: 10.1016/j.jpsychires.2013.03.018

Öngür, D., Drevets, W. C., and Price, J. L. (1998). Glial reduction in the subgenual prefrontal cortex in mood disorders. Proc. Natl. Acad. Sci. U.S.A. 95, 1329013295. doi: $10.1073 /$ pnas. 95.22 .13290

Pehrson, A. L., and Sanchez, C. (2015). Altered $\gamma$-aminobutyric acid neurotransmission in major depressive disorder: a critical review of the supporting evidence and the influence of serotonergic antidepressants. Drug Des. Devel. Ther. 9, 603-624. doi: 10.2147/DDDT.S62912

Pfleiderer, B., Michael, N., Erfurth, A., Ohrmann, P., Hohmann, U., Wolgast, M., et al. (2003). Effective electroconvulsive therapy reverses glutamate/glutamine deficit in the left anterior cingulum of unipolar depressed patients. Psychiatry Res. 122, 185-192. doi: 10.1016/S0925-4927(03)00003-9

Rajkowska, G. (2000). Dysfunction in neural circuits involved in the pathophysiology of mood disorders: postmortem studies in mood disorders indicate altered numbers of neurons and glial cells. Biol. Psychiatry 48, 766-777. doi: 10.1016/S0006-3223(00)00950-1

Rajkowska, G. (2002). Cell pathology in mood disorders. Semin. Clin. Neuropsychiatry 7, 281-292. doi: 10.1053/scnp.2002.35228

Rajkowska, G., and Miguel-Hidalgo, J. J. (2007). Gliogenesis and glial pathology in depression. CNS Neurol. Disord. Drug Targets 6, 219-233. doi: $10.2174 / 187152707780619326$

Rajkowska, G., and Stockmeier, C. A. (2013). Astrocyte pathology in major depressive disorder: insights from human postmortem brain tissue. Curr. Drug Targets 14, 1225-1236. doi: 10.2174/13894501113149990156

Rowland, L. M., Kontson, K., West, J., Edden, R. A., Zhu, H., Wijtenburg, S. A., et al. (2013). In vivo measurements of glutamate, GABA, and NAAG in schizophrenia. Schizophr. Bull. 39, 1096-1104. doi: 10.1093/schbul/sbs092

Sahlender, D. A., Savtchouk, I., and Volterra, A. (2014). What do we know about gliotransmitter release from astrocytes? Philos. Trans. R. Soc. Lond. B Biol. Sci. 369:20130592. doi: 10.1098/rstb.2013.0592

Salvadore, G., van der Veen, J. W., Zhang, Y., Marenco, S., Machado-Vieira, R., Baumann, J., et al. (2012). An investigation of amino-acid neurotransmitters as potential predictors of clinical improvement to ketamine in depression. Int. J. Neuropsychopharmacol. 15, 1063-1072. doi: 10.1017/S1461145711001593

Savitz, J. B., Price, J. L., and Drevets, W. C. (2014). Neuropathological and neuromorphometric abnormalities in bipolar disorder: view from the medial prefrontal cortical network. Neurosci. Biobehav. Rev. 42, 132-147. doi: 10.1016/j.neubiorev.2014.02.008

Schitine, C., Nogaroli, L., Costa, M. R., and Hedin-Pereira, C. (2015). Astrocyte heterogeneity in the brain: from development to disease. Front. Cell. Neurosci. 9:76. doi: $10.3389 /$ fncel.2015.00076

Schroeter, M. L., Steiner, J., Schönknecht, P., and Mueller, K. (2014). Further evidence for a role of $\mathrm{S} 100 \mathrm{~B}$ in mood disorders: a human gene expression mega-analysis. J. Psychiatr. Res. 53, 84-86. doi: 10.1016/j.jpsychires.2014.02.021

Sequeira, A., Mamdani, F., Ernst, C., Vawter, M. P., Bunney, W. E., Lebel, V., et al. (2009). Global brain gene expression analysis links glutamatergic and GABAergic alterations to suicide and major depression. PLoS ONE 4:e6585. doi: 10.1371/journal.pone. 0006585

Shende, V. H., McArthur, S., Gillies, G. E., and Opacka-Juffry, J. (2015). Astroglial plasticity is implicated in hippocampal remodelling in adult rats exposed to antenatal dexamethasone. Neural Plast. (in press). doi: 10.1155/2015/694347
Si, X., Miguel-Hidalgo, J. J., O’Dwyer, G., Stockmeier, C. A., and Rajkowska, G. (2004). Age-dependent reductions in the level of glial fibrillary acidic protein in the prefrontal cortex in major depression. Neuropsychopharmacology 29, 2088-2096. doi: 10.1038/sj.npp.1300525

Skolnick, P., Popik, P., and Trullas, R. (2009). Glutamate-based antidepressants: 20 years on. Trends Pharmacol. Sci. 30, 563-569. doi: 10.1016/j.tips.2009.09.002

Sofroniew, M. V., and Vinters, H. V. (2010). Astrocytes: biology and pathology. Acta Neuropathol. 119, 7-35. doi: 10.1007/s00401-009-0619-8

Steiner, J., Bogerts, B., Sarnyai, Z., Walter, M., Gos, T., Bernstein, H. G., et al. (2012). Bridging the gap between the immune and glutamate hypotheses of schizophrenia and major depression: potential role of glial NMDA receptor modulators and impaired blood-brain barrier integrity. World J. Biol. Psychiatry 13, 482-492. doi: 10.3109/15622975.2011.583941

Takasaki, C., Yamasaki, M., Uchigashima, M., Konno, K., Yanagawa, Y., and Watanabe, M. (2010). Cytochemical and cytological properties of perineuronal oligodendrocytes in the mouse cortex. Eur. J. Neurosci. 32, 1326-1336. doi: 10.1111/j.1460-9568.2010.07377.x

Teschemacher, A. G., Gourine, A. V., and Kasparov, S. (2015). A role for astrocytes in sensing the brain microenvironment and neuro-metabolic integration. Neurochem. Res. doi: 10.1007/s11064-015-1562-9 [Epub ahead of print]

Thompson, S. M., Kallarackal, A. J., Kvarta, M. D., Van Dyke, A. M., LeGates, T. A., and Cai, X. (2015). An excitatory synapse hypothesis of depression. Trends Neurosci. 38, 279-294. doi: 10.1016/j.tins.2015.03.003

Toro, C. T., Hallak, J. E., Dunham, J. S., and Deakin, J. F. (2006). Glial fibrillary acidic protein and glutamine synthetase in subregions of prefrontal cortex in schizophrenia and mood disorder. Neurosci. Lett. 404, 276-281. doi: 10.1016/j.neulet.2006.05.067

Uranova, N. A., Vostrikov, V. M., Orlovskaya, D. D., and Rachmanova, V. I. (2004). Oligodendroglial density in the prefrontal cortex in schizophrenia and mood disorders: a study from the Stanley Neuropathology Consortium. Schizophr. Res. 67, 269-275. doi: 10.1016/S0920-9964(03)00181-6

Van Horn, M. R., Sild, M., and Ruthazer, E. S. (2013). D-serine as a gliotransmitter and its roles in brain development and disease. Front. Cell. Neurosci. 7:39. doi: 10.3389 /fncel.2013.00039

Verkhratsky, A., Rodríguez, J. J., and Steardo, L. (2014). Astrogliopathology: a central element of neuropsychiatric diseases? Neuroscientist 20, 576-588. doi: $10.1177 / 1073858413510208$

Vostrikov, V. M., Uranova, N. A., and Orlovskaya, D. D. (2007). Deficit of perineuronal oligodendrocytes in the prefrontal cortex in schizophrenia and mood disorders. Schizophr. Res. 94, 273-280. doi: 10.1016/j.schres.2007.04.014

Walter, M., Henning, A., Grimm, S., Schulte, R. F., Beck, J., Dydak, U., et al. (2009). The relationship between aberrant neuronal activation in the pregenual anterior cingulate, altered glutamatergic metabolism, and anhedonia in major depression. Arch. Gen. Psychiatry 66, 478-486. doi: 10.1001/archgenpsychiatry.2009.39

Xu, J., Dydak, U., Harezlak, J., Nixon, J., Dzemidzic, M., Gunn, A. D., et al. (2013). Neurochemical abnormalities in unmedicated bipolar depression and mania: a 2D 1H MRS investigation. Psychiatry Res. 30, 235-241. doi: 10.1016/j.pscychresns.2013.02.008

Yüksel, C., and Öngür, D. (2010). Magnetic resonance spectroscopy studies of glutamate-related abnormalities in mood disorders. Biol. Psychiatry 68, 785794. doi: $10.1016 /$ j.biopsych.2010.06.016

Conflict of Interest Statement: The authors declare that the research was conducted in the absence of any commercial or financial relationships that could be construed as a potential conflict of interest.

Copyright (c) 2015 Bernstein, Meyer-Lotz, Dobrowolny, Bannier, Steiner, Walter and Bogerts. This is an open-access article distributed under the terms of the Creative Commons Attribution License (CC BY). The use, distribution or reproduction in other forums is permitted, provided the original author(s) or licensor are credited and that the original publication in this journal is cited, in accordance with accepted academic practice. No use, distribution or reproduction is permitted which does not comply with these terms. 